\title{
A Dimension Series for Multivariate Splines *
}

\author{
Louis J. Billera ${ }^{1}$ and Lauren L. Rose ${ }^{2}$ \\ ${ }^{1}$ Cornell University, Ithaca, NY 14853-7901, USA \\ ${ }^{2}$ Ohio State University, Columbus, OH 43210, USA
}

\begin{abstract}
For a polyhedral subdivision $\Delta$ of a region in Euclidean $d$-space, we consider the vector space $C_{k}^{r}(\Delta)$ consisting of all $C^{r}$ piecewise polynomial functions over $\Delta$ of degree at most $k$. We consider the formal power series $\sum_{k \geq 0} \operatorname{dim}_{\mathbb{R}} C_{k}^{r}(\Delta) \lambda^{k}$, and show, under mild conditions on $\Delta$, that this always has the form $P(\lambda) /(1-\lambda)^{d+1}$, where $P(\lambda)$ is a polynomial in $\lambda$ with integral coefficients which satisfies $P(0)=1$, $P(1)=f_{d}(\Delta)$, and $P^{\prime}(1)=(r+1) f_{d-1}^{\circ}(\Delta)$. We discuss how the polynomial $P(\lambda)$ and bases for the spaces $C_{k}^{r}(\Delta)$ can be effectively calculated by use of Gröbner basis techniques of computational commutative algebra. A further application is given to the theory of hyperplane arrangements.
\end{abstract}

\section{Introduction}

The concept of a piecewise polynomial function over a polyhedral subdivision of a region in Euclidean space is a fundamental one, rich with connections to many areas of mathematics, from very pure to extremely practical. By their very definition, the study of such functions involves both algebra and geometry, and, when smoothness conditions are imposed, also a suggestion of analysis. Perhaps the most important aspect involved in dealing with these objects is the interplay between the underlying combinatorics and geometry of the subdivision and the algebraic properties of the resulting set of functions. This interplay has received attention only recently [6], [7], [20].

The simplest form of this idea, that of a piecewise linear function over a simplicial subdivision, is well understood and much used, as is the case of general

* This research was partially supported by NSF Grants DMS-8403225 and DMS-8703370/DMS8896193. 
smooth piecewise polynomials over subdivisions in the line. The case of general continuous piecewise polynomials over higher-dimensional simplicial subdivisions can be successfully treated using a variety of methods (see [6], for example). However, serious difficulties already begin to arise in the case of planar simplicial subdivisions when we require global smoothness conditions on the functions. This case has only been attacked with a great deal of success in recent years [2], [3], [7]. Perhaps surprisingly, serious difficulties also arise in the continuous case, with general polyhedral subdivisions in two dimensions [11], [32].

Piecewise polynomial functions turn up in a variety of contexts. They are also called multivariate splines. They have for some time been used to approximate general smooth functions in the solution of partial differential equations by the socalled finite element method, as well as to interpolate scattered multidimensional data. More recently, they have come into play in computer-aided geometric design, computer graphics, and robotics as a means of providing an effective description of a surface for purposes of display and control. Current practice makes results even for two and three dimensions and low orders of smoothness to be of serious interest. At the current time, extremely little is known about the case of first-order smoothness in three dimensions [1].

For a (finite) polyhedral complex $\Delta$, let $C^{r}(\Delta)$ denote the set of all piecewise polynomial functions over $\Delta$ which are globally smooth of order $r$. If $C_{k}^{r}(\Delta)$ denotes the subset of $C^{r}(\Delta)$ consisting of functions involving only polynomials of degree at most $k$, then $C_{k}^{r}(\Delta)$ is a finite-dimensional vector space over the reals, and a fundamental problem in this area is to determine the dimension of this vector space as a function of known information about the subdivision $\Delta$. Of considerable practical interest is the associated problem of determining a basis for this space.

A serious difficulty with this problem is that the dimension of the space $C_{k}^{r}(\Delta)$ can depend not just on the combinatorics of the subdivision, but also on its "geometry," that is, how it is embedded in space. For example, triangulating a rectangle with two crossing diagonals results in the dimension of $C_{2}^{1}(\Delta)$ being one higher than the combinatorially identical triangulation in which the central point is not the intersection of the diagonals. (See [7] for a discussion of such "nongeneric" behavior and for further references.)

In light of this, then, what can we hope to say in general about the dimension of $C_{k}^{r}(\Delta)$ when only the combinatorial properties of $\Delta$ are considered? Surprisingly, perhaps, there is a great deal of invariance remaining if we consider the dimensions of all the $C_{k}^{r}(\Delta)$ 's as a whole. We study in this paper the generating function of the numbers $\operatorname{dim}_{\mathbb{R}} C_{k}^{r}(\Delta), k=0,1,2, \ldots$; that is, we consider the formal power series

$$
\sum_{k \geq 0} \operatorname{dim}_{\mathbb{R}} C_{k}^{r}(\Delta) \lambda^{k}
$$

We show (under mild restrictions on the subdivision $\Delta$ ) that this series (considered as an element of the ring of formal power series in $\lambda$ over $\mathbb{Z}$ ) always has the form

$$
\frac{P(\lambda)}{(1-\lambda)^{d+1}}
$$


where $P(\lambda)$ is a polynomial in $\lambda$ with integral coefficients and $d=\operatorname{dim}(\Delta)$. From this, it follows that, for large $k, \operatorname{dim}_{\mathbb{R}} C_{k}^{r}(\Delta)$ is given by a polynomial function of $k$. We further show that the polynomial $P(\lambda)$ always satisfies $P(0)=1, P(1)=f_{d}$, and $P^{\prime}(1)=(r+1) f_{d-1}^{\circ}$, where $f_{d}$ is the number of $d$-cells in the subdivision $\Delta$ and $f_{d-1}^{o}$ is the number of interior $(d-1)$-cells, both combinatorial invariants of $\Delta$. Thus while the individual numbers $\operatorname{dim}_{\mathbb{R}} C_{k}^{r}(\Delta)$ may change with changes in the geometry of $\Delta$ (in ways not yet well understood), the collection as a whole is rather more controlled; although the polynomial $P$ may vary as the embedding is changed, the three evaluations $P(0) P(1)$, and $P^{\prime}(1)$ will be unchanged. Except for [6], [7], and [20], the idea of considering all the spaces $C_{k}^{r}(\Delta)$ at once seems to have arisen only in [28] and [29].

A side benefit of this result is the observation that the entire series, being of the form $P(\lambda) /(1-\lambda)^{d+1}$, is determined by the polynomial $P(\lambda)$ and therefore can be calculated if $P(\lambda)$ can be calculated. This in fact can be done using Gröbner basis techniques of computational commutative algebra. We discuss this briefly here; a more extensive discussion can be found in [8].

To obtain the result described above, we make use of the fact that $C^{r}(\Delta)$, in addition to being a vector space, has the structure of a commutative ring under pointwise multiplication of functions, and so forms a module over the subring $R$ of global polynomial functions (i.e., those being given by the same polynomial on each cell of $\Delta$ ). In the remainder of this section we discuss this algebraic structure, and derive a completely algebraic criterion for a piecewise polynomial function to be $r$-fold smooth. In Section 2 we reduce the problem to the case in which each maximal cell of $\Delta$ contains the origin in $\mathbb{R}^{d}$. In this case, the resulting module is graded, and the series we are interested in is essentially the Hilbert series of this graded module. This gives us the form of the series as a rational function of $\lambda$, as well as the corollary that, for large values of $k$, the function $D(k)=\operatorname{dim}^{\mathbb{R}} C_{k}^{r}(\Delta)$ is given by a polynomial in $k$ of degree $d$ with leading coefficient $f_{d} / d$ !. In section 3 we consider, in a general setting, certain graded modules defined as the kernels of maps between free modules, and we derive some properties of their Hilbert functions. In Section 4 , we apply these results to the dimension series of the multivariate spline module, as well as to the Hilbert series of the Terao module (module of derivations) of a central arrangement of hyperplanes in a general vector space [18], [24], [35]. Finally, in Section 5, we discuss briefly the issue of computing the power series for the general modules defined in Section 3, as well as the required vector space bases.

Throughout this paper we use many basic results from commutative algebra. Good references for this material are [4] and [17].

We now introduce some preliminary notions. Let $\Delta$ be a polyhedral complex in $\mathbb{R}^{d}$, that is, a finite union of convex polytopes in $\mathbb{R}^{d}$ such that every face of a polytope in $\Delta$ is a face of the complex, and the intersection of any two faces of the complex is a face of each. Recall that, for a convex polytope $P$, a face $F \neq P$ is the intersection of $P$ with a hyperplane which does not meet the interior of $P$. Any such hyperplane will be called a supporting hyperplane for $F$. (See [12] for more details about polytopes and polyhedral complexes.) If $\Delta$ is any complex, the dimension of $\Delta$ is the maximum dimension of an element of $\Delta$. We say $\Delta \subset \mathbb{R}^{d}$ is pure if all maximal 
faces are of the same dimension. A special case of a polyhedral complex is a finite simplicial complex, where each face of $\Delta$ is a simplex.

By $d$-complex we will mean a pure $d$-dimensional polyhedral complex embedded in $\mathbb{R}^{d}$. In this case we may think of $\Delta$ as a partition of a region in $\mathbb{R}^{d}$ into finitely many $d$-polytopes. For a $d$-complex $\Delta$ and $i \leq d$, we denote the set of $i$-dimensional faces of $\Delta$ by $\Delta_{i}$, and the set of $i$-dimensional interior faces of $\Delta$ by $\Delta_{i}^{\circ}$. Similarly, $f_{i}(\Delta)$ denotes the number of $i$-dimensional faces of $\Delta$, and $f_{i}^{\circ}(\Delta)$ the number of $i$-dimensional interior faces of $\Delta$.

If $\Delta$ is simplicial, we can identify $\sigma \in \Delta$ with its set of vertices. Then $\tau \cup \sigma$ will correspond to the union of the vertex sets of $\tau$ and $\sigma$. If $\Delta$ is simplicial, we define the link of $\sigma$ in $\Delta$ by $\mathrm{lk}_{\Delta} \sigma \equiv\{\tau \in \Delta: \tau \cup \sigma \in \Delta, \tau \cap \sigma=\varnothing\}$, and the star of $\sigma$ in $\Delta$ by st $_{\Delta} \sigma \equiv\left\{\tau \cup \tau^{\prime}: \tau \in \mathrm{lk}_{\Delta} \sigma, \tau^{\prime} \subset \sigma\right\}$. For arbitrary complexes we define the star of $\sigma$ in $\Delta$ by

$$
\text { st }_{\Delta} \sigma \equiv\left\{\tau \in \Delta: \exists \tau^{\prime} \in \Delta \text { such that } \tau \subset \tau^{\prime} \text { and } \sigma \subset \tau^{\prime}\right\} \text {. }
$$

In other words, $\mathrm{st}_{\Delta} \sigma$ is the smallest subcomplex of $\Delta$ containing all faces which contain $\sigma$. If the complex $\Delta$ is understood, we will write st $\sigma$ or $1 \mathrm{k} \sigma$.

For a $d$-complex $\Delta$, we consider the graph $G(\Delta)$ with vertices corresponding to the elements of $\Delta_{d}$ and edges defined as follows: if $v, v^{\prime}$ are vertices of $G(\Delta)$ corresponding to $\sigma, \sigma^{\prime} \in \Delta_{d}$, then $\left\{v, v^{\prime}\right\}$ is an edge of $G(\Delta)$ if and only if $\sigma \cap \sigma^{\prime} \in \Delta_{d-1} . \Delta$ is said to be strongly connected if the graph $G(\Delta)$ is connected. A connected complex $\Delta$ is said to be hereditary if, for all $\sigma \in \Delta-\{\varnothing\}$, st $\sigma$ is strongly connected. (If $\Delta$ is simplicial, this is equivalent to the property that, for all $\sigma \in \Delta-\{\varnothing\}, \mathrm{k} \sigma$ is strongly connected.) From this condition it follows that $\Delta$ itself is strongly connected.

Let $\Delta$ be a $d$-complex and let $R=\mathbb{R}\left[x_{1}, \ldots, x_{d}\right]$, the polynomial ring over $\mathbb{R}$ in $d$ variables. We now define $C^{r}(\Delta)$ more explicitly and give an algebraic condition for smoothness.

Definition. If $r \in \mathbb{N}$ and $\Delta$ is a $d$-complex, then $C^{r}(\Delta)$ is the set of functions $F: \Delta \rightarrow \mathbb{R}$ such that:

(i) For all $\sigma \in \Delta_{d},\left.F\right|_{\sigma}$ is given by a polynomial in $R=\mathbb{R}\left[x_{1}, \ldots, x_{d}\right]$.

(ii) $F$ is continuously differentiable of order $r$.

In this definition, $F$ will be differentiable of order $r$ at a point $p$ in $\Delta$ if, for all $\sigma \in \Delta_{d}$ containing $p$, all partial derivatives of $\left.F\right|_{\sigma}$ up to order $r$ agree at $p$. Note that since $\sigma$ is $d$-dimensional, the polynomial $\left.F\right|_{\sigma}$ is uniquely determined. We will see that the differentiability condition on $F$ translates into a purely algebraic condition. This condition is used extensively in our study of $C^{r}(\Delta)$. We write $F_{\sigma}$ for $\left.F\right|_{\sigma}$.

For $\sigma \in \Delta$, let aff $\sigma$ denote the affine span of points in $\sigma$, and, for $f_{1}, \ldots, f_{n}$ in $R$, let $\left(f_{1}, \ldots, f_{n}\right)$ denote the ideal that they generate. If $T \subset R$ is any set of polynomials, we define the zero set of $T, Z(T) \equiv\left\{p \in \mathbb{R}^{d}: f(p)=0\right.$ for all $\left.f \in T\right\}$. If $X \subset \mathbb{R}^{d}$ is any set, we define the ideal of $X, I(X) \equiv\{f \in R: f(p)=0$ for all $p \in X\}$. Notice that $I$ and $Z$ are inclusion reversing, i.e. $X \subset Y$ implies $I(Y) \subset I(X)$, and $T \subset S$ implies $Z(S) \subset Z(T)$. We will need the following easily verified properties of $I$ and $Z$. 


\section{Proposition 1.1.}

(a) Let $A$ be an affine subspace of $\mathbb{R}^{d}$ of codimension c and let $A=H_{1} \cap \cdots \cap H_{c}$, where $H_{i}$ are hyperplanes in $\mathbb{R}^{d}$, and $l_{i}$ are affine forms such that $H_{i}=Z\left(l_{i}\right)$. Then $I(A)=\left(l_{1}, \ldots, l_{c}\right)$.

(b) If $\sigma$ is a convex polytope in $\mathbb{R}^{d}$, then $I(\sigma)=I(\mathrm{aff} \sigma)$ and $Z(I(\sigma))=$ aff $\sigma$.

Recall that for an ideal $I, I$ is the ideal generated by all $r$-fold products of elements of $I$.

Proposition 1.2 (Algebraic Criterion). Let $\Delta$ be a d-complex and let $F: \Delta \rightarrow \mathbb{R}$ be a piecewise polynomial function. Then $F \in C^{r}(\Delta)$ if and only if, for every pair of faces $\sigma_{1}, \sigma_{2}$ in $\Delta_{d}, F_{\sigma_{1}}-F_{\sigma_{2}}$ lies in $I\left(\sigma_{1} \cap \sigma_{2}\right)^{r+1}$.

Proof. Suppose $F \in C^{r}(\Delta)$. Then, for every pair of faces $\sigma_{1}, \sigma_{2}$ in $\Delta_{d}$, all partial derivatives up to order $r$ of $h \equiv F_{\sigma_{1}}-F_{\sigma_{2}}$ vanish on $\sigma_{1} \cap \sigma_{2}$. Then each of these lie in $I\left(\sigma_{1} \cap \sigma_{2}\right)$. If $\sigma_{1} \cap \sigma_{2}$ has codimension $c$, then by Proposition 1.1(a) there are $c$ hyperplanes defined by $c$ independent affine forms $l_{1}, \ldots, l_{c}$ such that $I\left(\sigma_{1} \cap \sigma_{2}\right)=$ $\left(l_{1}, \ldots, l_{c}\right)$. By an affine change of coordinates, we may assume $l_{i}=x_{i}$. Since $h \in I\left(\sigma_{1} \cap \sigma_{2}\right)$, we may write $h=r_{1} x_{1}+\cdots+r_{c} x_{c}$ where each $r_{i} \in R$. Let $i \leq c$. Then $\partial h / \partial x_{i}=\sum r_{j}\left(\partial x_{j} / \partial x_{i}\right)+\sum\left(\partial r_{j} / \partial x_{i}\right) x_{j} \quad$ where $j=1, \ldots, c$. Since $\partial h / \partial x_{i} \in I\left(\sigma_{1} \cap \sigma_{2}\right)$ and the second sum is also in $I\left(\sigma_{1} \cap \sigma_{2}\right)$, we get that $\sum r_{j}\left(\partial x_{j} / \partial x_{i}\right) \in I\left(\sigma_{1} \cap \sigma_{2}\right)$. Since $i \leq c$, this sum is just $r_{i}$. Thus each $r_{i} \in I\left(\sigma_{1} \cap \sigma_{2}\right)$, which shows $h \in I\left(\sigma_{1} \cap \sigma_{2}\right)^{2}$. We then can write $h=\sum r_{i j} x_{i} x_{j}$ where $i, j \leq C$. Taking second partials we show in the same way as before that $h \in I\left(\sigma_{1} \cap \sigma_{2}\right)^{3}$. Continuing this process we finally get that $h \in I\left(\sigma_{1} \cap \sigma_{2}\right)^{r+1}$, as desired.

Conversely, suppose that, for every pair of faces $\sigma_{1}, \sigma_{2}$ in $\Delta_{d}, F_{\sigma_{1}}-$ $F_{\sigma_{2}} \in I\left(\sigma_{1} \cap \sigma_{2}\right)^{r+1}$. Let $p \in \Delta$. If $p$ lies in $\sigma_{1}$ and $\sigma_{2}$, then, if $h=F_{\sigma_{1}}-F_{\sigma_{2}}$, we have $h(p)=0$, since $h \in I\left(\sigma_{1} \cap \sigma_{2}\right)^{r+1}$. We must show that all partial derivatives of $h$ up to order $r$ also vanish on $p$. As above we may assume $I\left(\sigma_{1} \cap \sigma_{2}\right)=\left(x_{1}, \ldots, x_{c}\right)$ where $c$ is the codimension of $\sigma_{1} \cap \sigma_{2}$. Then $I\left(\sigma_{1} \cap \sigma_{2}\right)^{r+1}$ is generated over $\mathbb{R}$ by monomials $x_{1}^{a_{1}} \cdots x_{d}^{a_{d}}$ where $a_{1}+a_{2}+\cdots+a_{c} \geq r+1$. The $i$ th partial derivative of any monomial of this form is either 0 or is the sum of terms of form $c x_{1}^{b_{1}} \cdots x_{d}^{b_{d}}$ where $c \in \mathbb{R}$ and $b_{1}+b_{2}+\cdots+b_{c} \geq r+1-i$. This will vanish at $p$ as long as $r+1-i \geq 1$, or $i \leq r$, so the same is true for $h$.

Remark and Notation. If $\sigma_{1} \cap \sigma_{2}=\tau \in \Delta_{d-1}$, then $I\left(\sigma_{1} \cap \sigma_{2}\right)=I(\tau)$ is principal and generated by an affine form, which we denote $l_{12}$ or $l_{\mathrm{r}}$. If $\sigma \in \Delta_{d}$, note that Proposition $1.1(b)$ implies $I(\sigma)=(0)$ since $\mathbb{R}$ is infinite.

The idea of the proof of Proposition 1.2 comes from Lemma 2.7 of [10]. We use this criterion as the definition of $C^{r}(\Delta)$ in all that follows. The following corollary can also be found in [7].

Corollary 1.3. If $\Delta$ is hereditary, then $F \in C^{r}(\Delta)$ if and only if, for every pair of faces $\sigma_{1}, \sigma_{2}$ in $\Delta_{d}$ which meet in ad -1 face $\tau, F_{\sigma_{1}}-F_{\sigma_{2}} \in I(\tau)^{r+1}=\left(l_{\tau}^{r+1}\right)$. 
Proof. Let $\sigma$ and $\sigma^{\prime}$ be in $\Delta_{d}$. Since $\Delta$ is hereditary, the star of $\sigma \cap \sigma^{\prime}$ is strongly connected. This means there is a sequence $\sigma=\sigma_{1}, \ldots, \sigma_{n}=\sigma^{\prime}$ in $\Delta_{d}$ where $\sigma_{i} \cap \sigma_{i+1}=\tau_{i} \in \Delta_{d-1}$ and each $\sigma_{i}$ is in the star of $\sigma \cap \sigma^{\prime}$, and so contains $\sigma \cap \sigma^{\prime}$. Then each $\tau_{i}$ also contains $\sigma \cap \sigma^{\prime}$. (It is possible that $\sigma \cap \sigma^{\prime}=\varnothing$.) We know $I(\tau) \subset I(\sigma)$ whenever $\sigma \subset \tau$. Then $I\left(\tau_{i}\right) \subset I\left(\sigma \cap \sigma^{\prime}\right)$ which implies

$$
F_{\sigma_{i}}-F_{\sigma_{i+1}} \in I\left(\tau_{i}\right)^{r+1} \subset I\left(\sigma \cap \sigma^{\prime}\right)^{r+1}
$$

and so

$$
F_{\sigma}-F_{\sigma^{\prime}}=F_{\sigma_{1}}-F_{\sigma_{n}}=\left(F_{\sigma_{1}}-F_{\sigma_{2}}\right)+\cdots+\left(F_{\sigma_{n-1}}-F_{\sigma_{n}}\right)
$$

lies in $I\left(\sigma \cap \sigma^{\prime}\right)^{r+1}$.

We now describe some important properties of $C^{r}(\Delta)$. Given an ordering $\sigma_{1}, \ldots, \sigma_{t}$ of the $d$-simplices of $\Delta, F \in C^{r}(\Delta)$ can be represented as a $t$-tuple of polynomials in $R$, i.e., $F=\left(f_{1}, \ldots, f_{t}\right)$, where each $f_{i}$ is just $F_{\sigma_{i}}$. In this way we see that $C^{r}(\Delta)$ is a submodule of $R^{d}$, the free $R$-module of rank $d$. The $R$-algebra structure of $C^{r}(\Delta)$ is given by

$$
\begin{aligned}
\left(f_{1}, \ldots, f_{t}\right)+\left(g_{1}, \ldots, g_{t}\right) & =\left(f_{1}+g_{1}, \ldots, f_{t}+g_{t}\right), \\
g \cdot\left(f_{1}, \ldots, f_{t}\right) & =\left(g f_{1}, \ldots, g f_{t}\right) \\
\left(f_{1}, \ldots, f_{t}\right) \cdot\left(g_{1}, \ldots, g_{t}\right) & =\left(f_{1} g_{1}, \ldots, f_{t} g_{t}\right)
\end{aligned}
$$

where $g \in R$ and $\left(f_{1}, \ldots, f_{t}\right),\left(g_{1}, \ldots, g_{t}\right)$ lie in $C^{r}(\Delta)$. To see, for example, that $\left(f_{1} g_{1}, \ldots, f_{t} g_{t}\right)$ is again an element of $C^{r}(\Delta)$, note that, for faces $\sigma_{i}$ and $\sigma_{j}$,

$$
f_{i} g_{i}-f_{j} g_{j}=f_{i}\left(g_{i}-g_{j}\right)+g_{j}\left(f_{i}-f_{j}\right)
$$

which lies in $I\left(\sigma_{i} \cap \sigma_{j}\right)^{r+1}$ by Proposition 1.2.

Recall that the rank of a module $M$ over $R$ is the dimension of $M \otimes_{R} F$ as a vector space of $F$, the quotient field of $R$, i.e., $F=\mathbb{R}\left(x_{1}, \ldots, x_{d}\right)$, the field of rational functions in $d$ variables over $\mathbb{R}$. The rank of $M$ is also the minimal number of $R$ linearly independent elements in $M$. (See [17].) $C^{r}(\Delta)$ has the following structure as an $R$-module.

Proposition 1.4. Let $\Delta$ be a d-complex. Then $C^{r}(\Delta)$ is a finitely generated torsion free $R$-module with rank $=f_{d}(\Delta)$.

Proof. Let $t=f_{d}(\Delta)$. The first two properties follow from the fact that $C^{r}(\Delta) \subset R^{t}$ and $R$ is a Noetherian integral domain. For the rank, we note that there exists a nonzero $q$ in $R$ with the property that $q R^{t} \subset C^{r}(\Delta)$. We can construct such a $q$ as follows. For each nonmaximal $\tau \in \Delta$, let $q_{\tau}$ be any nonzero element of $I(\tau)^{r+1}$. Now let $q=\prod q_{\tau}$. It is easy to verify that this $q$ works by using the algebraic criterion for 
$C^{r}(\Delta)$. We also have the inclusion $C^{r}(\Delta) \subset R^{t}$. Let $F$ be the quotient field of $R$. Since tensoring with $F$ is exact, the inclusions will yield injections of vector spaces over $F$,

$$
q R^{t} \otimes_{R} F \rightarrow C^{r}(\Delta) \otimes_{R} F \quad \text { and } \quad C^{r}(\Delta) \otimes_{R} F \rightarrow R^{t} \otimes_{R} F
$$

which become

$$
F^{t} \rightarrow C^{r}(\Delta) \otimes_{R} F \quad \text { and } \quad C^{r}(\Delta) \otimes_{R} F \rightarrow F^{t}
$$

using properties of tensor products. Since these are both injections, we must have $\operatorname{dim}_{F}\left(C^{r}(\Delta) \otimes_{R} F\right)=t$.

\section{Reduction to the Central Case}

Let $\Delta$ be a $d$-complex (a pure $d$-dimensional polyhedral complex embedded in $\mathbb{R}^{d}$ ). Let $D(k)$ denote the dimension of $C_{k}^{r}(\Delta)$ as a vector space over $\mathbb{R}$. Consider

$$
\sum_{k \geq 0} \operatorname{dim}_{\mathbb{R}} C_{k}^{r}(\Delta) \lambda^{k}
$$

the generating function of $D(k)$. In this section we will see that this series has the form of a polynomial of degree $d$. We do this by showing that $D(k)$ is the Hilbert function of a graded algebra, namely $C^{r}(\hat{\Delta})$, where $\hat{\Delta} \subset \mathbb{R}^{d+1}$ is the homogenization of $\Delta$. (For an introduction to the theory of generating functions, see [27].)

Let $\Delta \subset \mathbb{R}^{d}$ and $R=\mathbb{R}\left[x_{1}, \ldots, x_{d}\right]$. We define $\hat{\Delta} \subset \mathbb{R}^{d+1}$, the homogenization of $\Delta$, in the following manner. Embed $\Delta$ in $\mathbb{R}^{d+1}$ by sending $\left(a_{1}, \ldots, a_{d}\right)$ to $\left(a_{1}, \ldots, a_{d}, 1\right)$. Let $v=(0, \ldots, 0)$ in $\mathbb{R}^{d+1}$. Then let $\hat{\Delta}=\Delta \cdot v$, the join of $\Delta$ with $v$, which we define to be the complex $\Delta \cup\{\hat{\sigma}: \sigma \in \Delta\}$, where $\hat{\sigma}$ denotes the convex hull of $\sigma$ and $\{v\}$. See Fig. 1. Let $\hat{R}=R[z]$. Then $C^{r}(\hat{\Delta})$ is module over $\hat{R}$. If $f \in R$, we define the homogenization ${ }^{\mathrm{h}} f \in \hat{R}$ of $f$ by

$$
{ }^{\mathrm{h}} f\left(x_{1}, \ldots, x_{\mathrm{d}}, z\right)=z^{\partial f} f\left(x_{1} / z, \ldots, x_{d} / z\right),
$$

where $\partial f$ denotes the total degree of $f$. Similarly, if $F \in R^{t}$, we can define the homogenization of $F,{ }^{\mathrm{h}} F$, in $\hat{R}^{t}$ by

$$
{ }^{\mathrm{h}} F\left(x_{1}, \ldots, x_{d}, z\right)=z^{\partial F} F\left(x_{1} / z, \ldots, x_{d} / z\right) .
$$

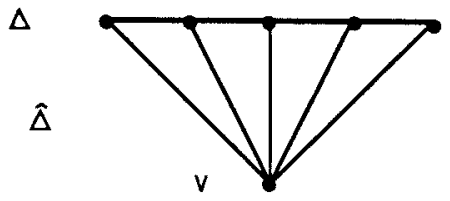

Fig. 1 
If $F=\left(f_{1}, \ldots, f_{t}\right)$, then $\partial F$ denotes the maximum of the $\partial f_{i}$ 's. It follows from the definitions that

$$
{ }^{\mathrm{h}} F={ }^{\mathrm{h}}\left(f_{1}, \ldots, f_{t}\right)=\left(\mathrm{z}^{\partial F-\partial f_{1}}\left({ }^{\mathrm{h}} f_{1}\right), \ldots, z^{\left.\partial F-\partial f_{\mathrm{t}}\left({ }^{\mathrm{h}} f_{t}\right)\right)}\right.
$$

Conversely, if $h \in \hat{R}$, we set $h(1)=h\left(x_{1}, \ldots, x_{d}, 1\right)$, and if $H=\left(h_{1}, \ldots, h_{t}\right) \in \hat{R}^{t}$, then $H(1)=\left(h_{1}(1), \ldots, h_{r}(1)\right)$. Let $I$ be an ideal in $R$. We define ${ }^{h} I$, the homogenization of $I$, to be the ideal in $\hat{R}$ generated by $\left\{{ }^{\mathrm{h}} f: f \in I\right\}$. The following properties of homogenization can be found in Section 5 of Chapter VII of [33].

Proposition 2.1. Let $F, G$ be in $R^{t}$, and let $I$ and $J$ be any ideals in $R$.

(a) ${ }^{\mathrm{h}}(F G)=\left({ }^{\mathrm{h}} F\right)\left({ }^{\mathrm{h}} G\right)$.

(b) $z^{\partial F+\partial G \mathrm{~h}}(F+G)=z^{\partial(F+G)}\left(z^{\partial G \mathrm{~h}} F+z^{\partial F \mathrm{~h}} G\right)$.

(c) ${ }^{\mathrm{h}} F(1)=F$.

(d) ${ }^{\mathrm{h}}(I J)={ }^{\mathrm{h}} I^{\mathrm{h}} J$.

Lemma 2.2. Let $I=I(\sigma)$ and $\hat{I}=I(\hat{\sigma})$. Then:

(1) $\left({ }^{\mathrm{h}} I\right)^{r}={ }^{\mathrm{h}}\left(I^{r}\right)$ for any $r$ in $\mathbb{N}$.

(2) $\hat{I}={ }^{h} I$.

Proof. (1) Follows from (d) above. For (2), we first note that if aff $(\sigma)=$ $Z\left(l_{1}, \ldots, l_{c}\right)$ in $\mathbb{R}^{d}$, then aff $(\hat{\sigma})=Z\left({ }^{\mathrm{h}} l_{1}, \ldots,{ }^{\mathrm{h}} l_{c}\right)$ in $\mathbb{R}^{d+1}$. Then $\hat{I}=\left({ }^{\mathrm{h}} l_{1}, \ldots,{ }^{\mathrm{h}} l_{c}\right)$ is clearly contained in ${ }^{\mathrm{h}} I$. Now let $f \in I$ and $p \in \hat{\sigma}$. Then $p=a(q, 1)+(1-a)(\overline{0}, 0)$ in $\mathbb{R}^{d} \times \mathbb{R}$, where $q \in \sigma$ and $0 \leq a \leq 1$. If $a \neq 0,{ }^{\mathrm{h}} f(p)={ }^{\mathrm{h}} f(a q, a)=a^{\partial f} f(q)=0$, since $f \in I$. If $a=0$, then $p$ is the origin and since ${ }^{\mathbf{b}} f$ is a nonconstant homogeneous polynomial (since $I \neq R$ ), ${ }^{\mathrm{h}} f(p)=0$. This shows ${ }^{\mathrm{h}} f \in \hat{I}$.

Lemma 2.3. If $F \in C^{r}(\Delta)$, then ${ }^{\mathrm{h}} F \in C^{r}(\hat{\Delta})$.

Proof. Let $\sigma_{1}, \ldots, \sigma_{\ell}$ be an ordering of $\Delta_{d}$, where $t=f_{d}(\Delta)$. Let $F=\left(f_{1}, \ldots, f_{t}\right)$ with respect to this ordering. By definition,

$$
{ }^{\mathrm{h}} F={ }^{\mathrm{h}}\left(f_{1}, \ldots, f_{t}\right)=\left(z^{\partial F-\partial f_{1}}\left({ }^{\mathrm{h}} f_{1}\right), \ldots, z^{\left.\partial F-\partial f_{\mathrm{t}}\left({ }^{\mathrm{h}} f_{\mathrm{t}}\right)\right)}\right.
$$

Let $f=f_{i}, g=f_{j}$, and $I=I\left(\sigma_{i} \cap \sigma_{j}\right)$. By the algebraic criterion for $C^{r}(\Delta)$ (Proposition 1.2), $f-g \in I^{r+1}$. Then

$$
\begin{aligned}
& z^{\partial F-\partial f}\left({ }^{\mathrm{h}} f\right)-z^{\partial F-\partial g}\left({ }^{\mathrm{h}} g\right)=z^{\partial F-\partial f-\partial g}\left(z^{\partial g}\left({ }^{\mathrm{h}} f\right)-z^{\partial f}\left({ }^{\mathrm{h}} g\right)\right) \\
& =z^{\partial F-\partial(f+g) \mathbf{b}}(f-g) \in{ }^{\mathrm{h}}\left(I^{r+1}\right)=\left({ }^{\mathrm{h}} I\right)^{r+1}=\hat{I}^{r+1}
\end{aligned}
$$

by Proposition 2.1(b) and Lemma 2.2, since $f-g \in I^{r+1}$. Now by the algebraic criterion for $C^{r}(\hat{\Delta})$, this implies that ${ }^{\mathrm{h}} F \in C^{r}(\hat{\Delta})$.

Let $A$ be a graded $\mathbb{R}$-algebra. Then $A=A_{0} \oplus A_{1} \oplus \cdots$, where $A_{0}=\mathbb{R}$, and $A_{i} A_{j} \subset A_{i+j}$. We will show that $C^{r}(\hat{\Delta})$ is a graded algebra, and that $C_{k}^{r}(\Delta)$ is 
isomorphic, as a vector space, to $\left[C^{r}(\hat{\Delta})\right]_{k}$. More generally, we make the following definition.

Definition 2.4. Let $\Delta$ be a $d$-complex. We say $\Delta$ is central if there is some vertex $v_{0}$ in $\Delta$ such that every $\sigma$ in $\Delta_{d}$ contains $v_{0}$. (Note that $\hat{\Delta}$ is always central.)

Lemma 2.5. Let $\Delta$ be a central d-complex. Then $C^{r}(\Delta)$ is a graded $\mathbb{R}$-algebra.

Proof. Let $v_{0}$ be the vertex which lies on every face of $\Delta_{d}$. Without loss of generality, we may assume that $v_{0}$ is the origin. Then, for any $\sigma$ in $\Delta, I(\sigma)$ and $I(\sigma)^{r+1}$ are both homogeneous ideals, since $I(\sigma)$ is generated by affine forms (see Proposition 1.1(a)) which must vanish at the origin.

Let $F=\left(f_{1}, \ldots, f_{t}\right)$ be in $C^{r}(\Delta)$ and let $F_{k}=\left(f_{1 k}, \ldots, f_{t k}\right)$ be the homogeneous component of $F$ of degree $k$. To show $C^{r}(\Delta)$ is graded it is sufficient to show that $F_{k}$ is in $C^{r}(\Delta)$. Let $I_{i j}=I\left(\sigma_{i} \cap \sigma_{j}\right)^{r+1}$. Recall that if $F$ is in $C^{r}(\Delta)$, then, for all $i$ and $j$, $f_{i}-f_{j} \in I_{i j}$ which is homogeneous. Then all homogeneous components of $f_{i}-f_{j}$ lie in $I_{i j}$, so $f_{i k}-f_{j k}=\left(f_{i}-f_{j}\right)_{k} \in I_{i j}$. Thus $F_{k} \in C^{r}(\Delta)$.

Theorem 2.6. $\left[C^{r}(\hat{\Delta})\right]_{k} \cong C_{k}^{r}(\Delta)$ as $\mathbb{R}$-vector spaces.

Proof. Define a map

$$
\varphi:\left[C^{r}(\hat{\Delta})\right]_{k} \longrightarrow C_{k}^{r}(\Delta)
$$

by $\varphi(H)=H(1) . \varphi$ is clearly $\mathbb{R}$-linear. Let $H=\left(h_{1}, h_{2}, \ldots, h_{t}\right)$, where $h_{i} \in R[z]$, $t=f_{\mathrm{d}}(\Delta)$, and $\sigma_{1}, \ldots, \sigma_{t}$ is an ordering of $\Delta_{\mathrm{d}}$. Let $I_{i j}=I\left(\sigma_{i} \cap \sigma_{j}\right)^{r+1}$. We must show that $\varphi$ is well defined and is an isomorphism of vector spaces over $\mathbb{R}$.

$\varphi(H) \in C_{k}^{r}(\Delta):$ If $H=\left(h_{1}, h_{2}, \ldots, h_{t}\right) \in\left[C^{r}(\hat{\Delta})\right]_{k}$ and $h_{i}-h_{j} \in{ }^{h} I_{i j}$, then $h_{i}(1)-$ $h_{j}(1)=\left(h_{i}-h_{j}\right)(1)$ which lies in $I_{i j}$ by Proposition 2.1(c), so $H(1) \in C_{k}^{r}(\Delta)$.

$\varphi$ is injective: If $H(1) \equiv 0$, then $h_{i}(1) \equiv 0$ for all $i$. This implies $z-1$ divides $h_{i}$ for all $i$. Then $h_{i} \equiv 0$ for all $i$, since each $h_{i}$ is homogeneous. This implies $H \equiv 0$.

$\varphi$ is surjective: Let $F \in C_{k}^{r}(\Delta)$ have degree $n \leq k$. By Lemma $2.3,{ }^{\text {h }} F \in C^{r}(\hat{\Delta}) .{ }^{\text {h }} F$ has degree $n$, so $z^{k-n}\left({ }^{h} F\right)$ will be in $\left[C^{r}(\widehat{\Delta})\right]_{k}$, and $\left(z^{k-n}\left({ }^{h} F\right)\right)(1)=F$ using Proposition 1(c). This proves surjectivity and the theorem.

We now define Hilbert series for graded modules and relate this to the central hereditary case. By Theorem 2.6, it follows that $\sum \operatorname{dim}_{\mathbb{R}} C_{k}^{r}(\Delta) \lambda^{k}$ is the Hilbert series of $C^{r}(\hat{\Delta})$ so we can apply the general theory about Hilbert series to the dimension series above.

Let $R=\mathbb{R}\left[x_{1}, \ldots, x_{d}\right]$. If $R_{i}$ consists of all homogeneous polynomials of degree $i$, then $R=R_{0} \oplus R_{1} \oplus \cdots$ is a graded $\mathbb{R}$-algebra. Let $M$ be a finitely generated $\mathbb{N}$-graded $R$-module, i.e., $M=\oplus M_{i}$ where $R_{i} M_{j} \subset M_{i+j}$. Since $R$ is a polynomial ring, (Krull) $\operatorname{dim} R=d$, the number of variables. Recall that $\operatorname{dim} M \equiv \operatorname{dim} R / I$, where $I=$ ann $M$, the annihilator of $M$. The codimension of $M, \operatorname{codim} M \equiv$ $d$-dim $M$. The Hilbert function of $M$ is defined by $H(M, i)=\operatorname{dim}_{\mathbb{R}} M_{i}$. Since $M$ is finitely generated and $R$ is finitely generated as an $\mathbb{R}$-algebra, this function will take 
values in $\mathbb{N}$. The Hilbert series of $M$, which we denote $\mathscr{F}(M, \lambda)$, is the power series $\sum H(M, i) \lambda^{i}$ in $\mathbb{N}[[\lambda]]$. If $M$ is a finitely generated graded $R$-module, then, for sufficiently large $i, H(M, i)$ is given by a polynomial with rational coefficients of degree $(\operatorname{dim} M)-1$. This polynomial is called the Hilbert polynomial of $M$. (See Theorem 7.5 of [13] or [26].)

The next proposition follows from Theorem 11 and Corollary 11.2 of [4].

Proposition 2.7. If $M$ is finitely generated then:

(a) $\mathscr{F}(M, \lambda)$ has the forms:

(i) $P(M, \lambda) /(1-\lambda)^{d}$ where $P(M, \lambda) \in \mathbb{Z}[\lambda]$ and $d=\operatorname{dim} R$.

(ii) $Q(M, \lambda) /(1-\lambda)^{s}$ where $Q(M, \lambda) \in \mathbb{Z}[\lambda]$ and $s=\operatorname{dim} M$. In this case, $(1-\lambda)$ does not divide $Q$.

(b) Let $Q(M, \lambda)=\sum_{j=0}^{\operatorname{deg} Q} a_{j} \lambda^{j}$. Then the Hilbert polynomial of $M$ is

$$
\sum_{j=0}^{\operatorname{deg} Q} a_{j}\left(\begin{array}{c}
d+i-j-1 \\
d-1
\end{array}\right) .
$$

Since $C^{r}(\hat{\Delta})$ is torsion free (by Proposition 1.4), its annihilator is zero, so the Krull dimension of $C^{r}(\hat{\Delta})$ is $d+1$. The above together with Theorem 2.6 gives

Theorem 2.8. For any d-complex, we always have

$$
\sum_{k \geq 0} \operatorname{dim}_{\mathbb{R}} C_{k}^{r}(\Delta) \lambda^{k}=P(\lambda) /(1-\lambda)^{d+1},
$$

where $P(\lambda)=P\left(C^{r}(\hat{\Delta}), \lambda\right)$ is a polynomial in $\lambda$ with integer coefficients. Further, if $D(k)=\operatorname{dim}_{\mathbb{R}} C_{k}^{r}(\Lambda)$, then, for large $k, D(k)$ is a polynomial of degree $d$ with rational coefficients; in fact, if $P(\lambda)=\sum_{j=0}^{\operatorname{deg} P} a_{j} \lambda^{j}$, then, for large $k$,

$$
D(k)=\sum_{j=0}^{\operatorname{deg} P} a_{j}\left(\begin{array}{c}
d+i-j \\
d
\end{array}\right) .
$$

Note that the leading coefficient of $D(k)$ is $P(1) / d$ !. For hereditary complexes, it will follow from Theorem 4.5 that this leading coefficient is $f_{d} / d$ !.

\section{Modules Defined by Maps Between Free Modules}

In this section $R=K\left[x_{1}, \ldots, x_{d}\right]$, the polynomial ring over $K$ in $d$ variables, where $K$ is any field. We investigate the Hilbert series of $M$, where $M$ is a graded $R$ module which arises as the kernel of a map between free $R$-modules. In particular, we study the polynomial $P(M, \lambda)$ from the previous section in the case of modules defined by maps between free modules. In Section 4 we will apply these results to splines and hyperplane arrangements.

If $M$ is any finitely generated graded $R$-module and $s \in \mathbb{N}$, we define $M(-s), M$ shifted by $s$, to be equal to $M$ as an $R$-module, but with grading $[M(-s)]_{i}=M_{i-s}$ where we set $M_{j}=0$ if $j<0$. If $M$ is graded by $\mathbb{N}$, then so is $M(-s)$. It follows that 
$H(M(-s), i)=H(M, i-s)$, so multiplying by $\lambda^{i}$ and summing over $i$ we get $\mathscr{F}(M(-s), \lambda)=\lambda^{s} \mathscr{F}(M, \lambda)$. Notice that $\mathscr{F}\left(M^{n}, \lambda\right)=n \mathscr{F}(M, \lambda)$.

If $M$ and $N$ are graded $R$-modules, then $\varphi: M \rightarrow N$ is a homogeneous map (of degree 0 ) if $\varphi$ is an $R$-linear map and $\varphi\left(M_{i}\right) \subset N_{i}$ for all $i$. It is easy to see that the kernel and image of a homogeneous map are also graded with grading $(\operatorname{ker} \varphi)_{i}=$ $M_{i} \cap \operatorname{ker} \varphi$, and $(\operatorname{Im} \varphi)_{i}=\varphi\left(M_{i}\right)$. The cokernel of $\varphi$ is also graded with grading $(\operatorname{coker} \varphi)_{i}=N_{i} / \varphi\left(M_{i}\right)$. If

$$
0 \longrightarrow M_{n} \longrightarrow \cdots \longrightarrow M_{1} \longrightarrow M_{0} \longrightarrow 0
$$

is an exact sequence of graded $R$-modules and homogeneous maps, then for any $i \in \mathbb{N}$

$$
0 \longrightarrow\left[M_{n}\right]_{i} \longrightarrow \cdots \longrightarrow\left[M_{1}\right]_{i} \longrightarrow\left[M_{0}\right]_{i} \longrightarrow 0
$$

is an exact sequence of vector spaces over $K$, so

$$
H\left(M_{0}, i\right)-H\left(M_{1}, i\right)+\cdots+(-1)^{n} H\left(M_{n}, i\right)=0 .
$$

Multiplying by $\lambda^{i}$ and summing with respect to $i$ we get

$$
\mathscr{F}\left(M_{0}, \lambda\right)-\mathscr{F}\left(M_{1}, \lambda\right)+\cdots+(-1)^{n} \mathscr{F}\left(M_{n}, \lambda\right)=0 .
$$

We now state some preliminary results. Let $S$ be an arbitrary Noetherian ring. The height of an ideal $I$ is defined to be the minimum of the heights of the prime ideals containing $I$. The next lemma follows easily from the definitions.

Lemma 3.1. Let $S$ be a Noetherian ring and let $I$ be any ideal in $S$. Then

$$
\text { height } I+\operatorname{dim}(S / I) \leq \operatorname{dim} S \text {. }
$$

If $M$ is a module over a Noetherian ring $S$, then $\theta_{1}, \ldots, \theta_{k}$ in $S$ is an $M$-regular sequence (or $M$-sequence) if $\left(\theta_{1}, \ldots, \theta_{k}\right) M \neq M$ and, for $1 \leq i \leq k, \theta_{i}$ is not a zero divisor on $M /\left(\theta_{1}, \ldots, \theta_{i-1}\right) M$. If $M=S$, we say $\theta_{1}, \ldots, \theta_{k}$ is a reguar sequence (or $S$-sequence). The grade of an ideal $I$ is the maximal length of an $S$-sequence contained in $I$. The following is Theorem 132 in [14].

Proposition 3.2. Let $S$ be a Noetherian ring and let $I$ an ideal in $S$. Then height $I \geq$ grade $I$.

The next lemma follows immediately from Lemma 3.1 and Proposition 3.2.

Lemma 3.3. Let $S$ be a Noetherian ring, let $N$ be any $S$-module, and let $I=$ ann $N$. Then $\operatorname{codim} N \geq$ grade $I$. 
Recall that $R=K\left[x_{1}, \ldots, x_{d}\right]$. Let $n$ and $t$ be in $\mathbb{N}$, where $n \geq 2$. We will consider maps from $R^{t+n}$ to $R^{n}$ given by $n \times(t+n)$ matrices of the form

$$
A=(B \mid C)
$$

satisfying the following conditions:

(i) $B$ is an $n \times t$ matrix with entries in $K$.

(ii) $C$ is an $n \times n$ diagonal matrix whose diagonal entries, $f_{i}(i=1, \ldots, n)$, are powers of homogeneous, (nonzero) irreducible polynomials, $l_{i}(i=$ $1, \ldots, n)$, at least two of which are distinct.

(iii) For all $i$, there is a column $B(i)$ in $B$ whose $i$ th entry is nonzero and, for every $j \neq i$ such that $\left\{f_{i}, f_{j}\right\}$ are not relatively prime, the $j$ th entry is equal to 0 .

Let $M=$ kernel of $A, N=$ cokernel of $A$, and $I=$ ann $N$. Then we have an exact sequence:

$$
0 \longrightarrow M \longrightarrow \mathrm{R}^{\mathrm{t}+\mathrm{n}} \stackrel{A}{\longrightarrow} R^{n} \longrightarrow N \longrightarrow 0
$$

The main result of this section is that under these conditions, $P^{\prime}(M, 1)$ is completely determined by the degrees of $f_{1}, \ldots, f_{k}$, where $P^{\prime}$ denotes the derivative of $P$ with respect to $\lambda$. Recall that the rank of a module $M$ over $R$ is $\operatorname{dim}_{F} M \otimes_{R} F$, where $F$ is the quotient field of $R$. As before, by localizing the above sequence at $(0)$, we can show that $M$ has rank $t$.

Proposition 3.4. Under the conditions outlined above, $M$ and $N$ are graded $R$ modules, and $P(M, 1)=t, P(N, 1)=0$.

Proof. Let $d_{j}=$ degree of $f_{j}$. Then the first $t$ columns of $A$ lie in $K^{n}=\left(R_{0}\right)^{n}$, and column $t+j$ lies in $\left(R\left(-d_{j}\right)\right)^{n}$, for $1 \leq j \leq n$, so we can view $A$ as a homogeneous map of degree 0 from $R^{t} \oplus\left(\oplus{ }_{j=1}^{n} R\left(-d_{j}\right)\right)$ to $R^{n}$. Then $M$ and $N$ are graded with gradings mentioned above. We now have an exact sequence of graded modules and degree 0 maps

$$
0 \longrightarrow M \longrightarrow \mathrm{R}^{\mathbf{l}} \oplus\left(\bigoplus_{j=1}^{n} R\left(-d_{j}\right)\right) \stackrel{A}{\longrightarrow} R^{n} \longrightarrow N \longrightarrow 0
$$

and so

$$
\mathscr{F}(M, \lambda)+n \cdot \mathscr{F}(R, \lambda)=t \cdot \mathscr{F}(R, \lambda)+\sum \mathscr{F}\left(R\left(-d_{j}\right), \lambda\right)+\mathscr{F}(N, \lambda)
$$

Since $1 /(1-\lambda)^{d}=\sum_{i \geq 0}\left(\begin{array}{c}d+i-1 \\ d-1\end{array}\right) \lambda^{i}$ and $\left(\begin{array}{c}d+i-1 \\ d-1\end{array}\right)$ is the number of monomials in $K\left[x_{1}, \ldots, x_{\mathrm{d}}\right]$ of degree $i$ and also the dimension of $R_{i}$ over $K$, we see that 
$\mathscr{F}(R, \lambda)=1 /(1-\lambda)^{d}$ and $\mathscr{F}\left(R\left(-d_{j}\right), \lambda\right)=\lambda^{d_{j}} /(1-\lambda)^{d}$. Using this and multiplying both sides of $(3.5)$ by $(1-\lambda)^{d}$, we get

$$
P(M, \lambda)=t-n+\sum_{j=1}^{n} \lambda^{d_{j}}+P(N, \lambda),
$$

where $P$ is as in Proposition 2.7.

Now det $C$ is a nonzero element of $I=$ ann $N$, which implies that grade $I \geq 1$ since $R$ is a domain, and so, by Lemma 3.3, codim $N \geq 1$. By Proposition 2.7, this implies $1-\lambda$ divides $P(N, \lambda)$. Substituting 1 for $\lambda$ in the equation above, we get $P(M, 1)=t$.

Remark. In fact, if $M$ is any finitely generated graded $R$-module, $P(1)=\operatorname{rank} M$. However, the benefit of the preceding proof is that we have a formula connecting the Hilbert series of $M$ and $N$. We state this in a corollary.

Corollary 3.6. Let $d_{j}=\operatorname{deg} f_{j}$. Then $P(M, \lambda)=t-n+\sum_{j=1}^{n} \lambda^{d_{j}}+P(N, \lambda)$.

In Section 5 we will discuss methods of computing the Hilbert series of $M$. Using this corollary, we reduce the problem to that of computing the Hilbert series of $N$. In fact, since $N=R^{n} / \operatorname{Im} A$, it is sufficient to compute the Hilbert series of $\operatorname{Im} A$, the submodule of $R^{n}$ generated by the columns of $A$.

The following lemma is the crux of the proof of Theorem 3.9.

Lemma 3.7. If $d \geq 2$, then $\operatorname{codim} N \geq 2$.

Proof. By Lemma 3.3, it is sufficient to show that grade $I \geq 2$, where $I=$ ann $N$. To show this, we must find an $R$-sequence (regular sequence) of length 2 in $I$. Notice that

$$
r \in I \text { if and only if } r e_{i} \in \operatorname{Im} A \text { for all } 1 \leq i \leq n \text {. }
$$

Here $e_{1}, \ldots, e_{n}$ are the standard basis vectors for $R^{n}$. In particular, $f_{i} e_{i} \in \operatorname{Im} A$ since it is a column of $A$. Let $Q$ be the least common multiple of the $f_{i}$ 's. Let $\Gamma \subset\left\{f_{1}, f_{2}, \ldots, f_{n}\right\}$ be a set of minimal cardinality with the property that $Q=$ $\prod_{f \in \Gamma} f$. Such a set exists by condition (ii) above. Notice that, for any pair $f_{i}, f_{j}$ in $\Gamma$ with $i \neq j, f_{i}$ and $f_{j}$ are relatively prime.

Claim 1. $Q / f \in I$ for all $f \in \Gamma$.

Proof. Let $f \in \Gamma$. By (3.8), we must show that $(Q / f) e_{i} \in \operatorname{Im} A$ for all $i$. By condition (iii), for any $i$ there is a column $B(i)=\left(b_{1}, \ldots, b_{n}\right)^{\mathrm{T}}$ in $B$ such that $b_{i} \neq 0$ and if $j \neq i$ and $\operatorname{gcd}\left(f_{i}, f_{j}\right) \neq 1, b_{j}=0$. Recall that $b_{i} \in K$ so $B(i) / b_{i} \in \operatorname{Im} A$. Thus we may assume without loss of generality that $b_{i}=1$. If $f$ and $f_{i}$ are relatively prime, 
then $f_{i}$ divides $Q / f$ and so $(Q / f) e_{i}=\left(Q / f f_{i}\right)\left(f_{i} e_{i}\right) \in \operatorname{Im} A . \operatorname{If} \operatorname{gcd}\left(f, f_{i}\right) \neq 1$, we may write

$$
(Q / f) e_{i}=r_{0} B(i)+\sum_{j \neq i} r_{j}\left(f_{j} e_{j}\right)
$$

where $r_{0}=Q / f$ and $r_{j}=-b_{j}\left(Q / f f_{j}\right)$. Clearly, $r_{0} \in R$. Suppose $j \neq i$. We must show that $r_{j} \in R$. If $\operatorname{gcd}\left(f_{i}, f_{j}\right) \neq 1$, then $b_{j}=0$, so $r_{j}=0 \in R$. Suppose $\operatorname{gcd}\left(f_{i}, f_{j}\right)=1$. Then if $l$ is the irreducible polynomial corresponding to $f$, condition (ii) implies $l_{i} \neq c l_{j}$ for any $c$ in $K$. We have $l=c l_{i}$ for some $c \neq 0$ in $K, \operatorname{since} \operatorname{gcd}\left(f, f_{i}\right) \neq 1$. This implies $l \neq c l_{j}$ for any $c$, and so $\operatorname{gcd}\left(f, f_{j}\right)=1$. Then $Q / f f_{j} \in R$, so $r_{j} \in R$, proving the claim.

Let

$$
Q^{*}=\sum_{f \in \Gamma}(Q / f)
$$

Claim 2. If $Q R+Q^{*} R \neq R$, then $Q$ and $Q^{*}$ form an $R$-sequence in $I$.

Proof. Since $Q / f \in I$ for all $f \in \Gamma, Q$ and $Q^{*}$ both lie in $I . Q \neq 0$ by construction, and, since $R$ is a domain, this means $Q$ is not a zero-divisor in $R$. Let $g$ in $R$ be such that $Q^{*} g \equiv 0$ in $R /(Q)$. This means there exists $h \in R$ such that $Q^{*} g=h Q$. Let $f \in \Gamma$. Then $f \mid Q$ so $f \mid Q^{*} g$. We also have $f \mid\left(Q / f_{i}\right)$ whenever $f_{i} \in \Gamma-\{f\}$. This implies $f \mid(Q / f) g$, but $f$ and $Q / f$ are relatively prime so $f \mid g$. This implies $Q \mid g$ and hence $g \equiv 0$. This shows $Q^{*}$ is not a zero divisor $\bmod Q$. Finally, since by hypothesis $Q R+Q^{*} R \neq R, Q$ and $Q^{*}$ form an $R$-sequence in $I$.

If $Q R+Q^{*} R=R$, then $Q, Q^{*} \in I$ implies that $I=R$. In this case, since $R=K\left[x_{1}, \ldots, x_{d}\right]$ and $d \geq 2, x_{1}$ and $x_{2}$ form an $R$-sequence of length 2 . This proves the lemma.

Note. We did not use the condition $d \geq 2$ until the end of the previous proof, so in particular, Claim 2 is true when $d=1$.

We are now ready to prove the theorem.

Theorem 3.9. If $A$ is a matrix satisfying (i)-(iii) and $M=\operatorname{ker} A$, then

$$
P^{\prime}(M, 1)=\sum_{j=1}^{n} \operatorname{deg}\left(f_{j}\right)
$$

Proof. We have the formula

$$
P(M, \lambda)=t-n+\sum_{j=1}^{n} \lambda^{d_{j}}+P(N, \lambda)
$$


where $d_{j}=\operatorname{deg}\left(f_{j}\right)$. By Lemma 3.7, if $d \geq 2$, then $\operatorname{codim} N \geq 2$, which means that $(1-\lambda)^{2}$ divides $P(N, \lambda)$. Taking the first derivative and evaluating at 1 , we get $P^{\prime}(M, 1)=\sum d_{j}$. If $d=1$, grade $I=1$ so $I$ cannot contain an $R$-sequence of length 2. Then Claim 2 from the proof of Lemma 3.7 implies that $Q R+Q^{*} R=R$, and, since $Q, Q^{*} \in I$, we must have $I=R$. Then $N$ must be 0 , so $P(N, \lambda)=0$.

Remark. We have shown that if $d=1$ (or $A$ is surjective), then

$$
P(M, \lambda)=t-n+\sum_{j=1}^{n} \lambda^{d_{j}}
$$

\section{Hilbert Series of Splines and of Hyperplane Arrangements}

We now apply the results of the previous section to splines and hyperplane arrangements. We show that for both of these applications, the modules studied can be realized as the kernel of a map between free modules which satisfies conditions (i)-(iii) from Section 3. In the case of splines, we must restrict ourselves to hereditary complexes.

We first define a matrix which we can associate to $C^{r}(\Delta)$ when $\Delta$ is hereditary. This matrix will be a useful tool in the study of $C^{r}(\Delta)$.

If $\Delta$ is hereditary $d$-complex, we can view $C^{r}(\Delta)$ as the kernel of a map between free $R$-modules. We show this as follows. Recall the definition of the graph $G(\Delta)$, with vertices and edges corresponding to elements of $\Delta_{d}$ and $\Delta_{d-1}^{\circ}$, respectively. (See Section I.)

Definition 4.1. Given an ordering of $\Delta_{d}$ and $\Delta_{d-1}^{\circ}$, we define the boundary matrix of $\Delta, \partial(\Delta)$, to be the transpose of the node-arc incidence matrix of $G(\Delta)$, i.e.,

$$
\partial(\Delta)=\left(a_{i j}\right)=\left\{\begin{aligned}
1 & \text { if } v_{j} \text { is the smaller vertex of } e_{i} \\
-1 & \text { if } v_{j} \text { is the larger vertex of } e_{i} \\
0 & \text { otherwise. }
\end{aligned}\right.
$$

Definition 4.2. If $\Delta$ is hereditary, we define the matrix associated to $C^{r}(\Delta), A(\Delta, r)$, to be

$$
\left[\begin{array}{l|lll} 
& \tilde{l}_{1} & & \\
& & \ddots & \\
& & \tilde{l}_{n}
\end{array}\right]
$$

where $\partial=\partial(\Delta), n=f_{d-1}^{\circ}$, and, if $\sigma_{i} \in \Delta_{d-1}^{\circ}, l_{i}$ is the form defining $\sigma_{i}$ and $\tilde{l}_{i}=l_{i}^{r+1}$. (Note that the right-hand section is a diagonal matrix.) Let $M(\Delta, r)$ denote the kernel of $A(\Delta, r)$. It is clear that $M(\Delta, r)$ does not depend on the ordering of $\Delta_{d-1}^{\circ}$.

Proposition 4.3. If $\Delta$ is hereditary, then, for any ordering of $\Delta_{d}, C^{r}(\Delta) \cong M(\Delta, r)$. 


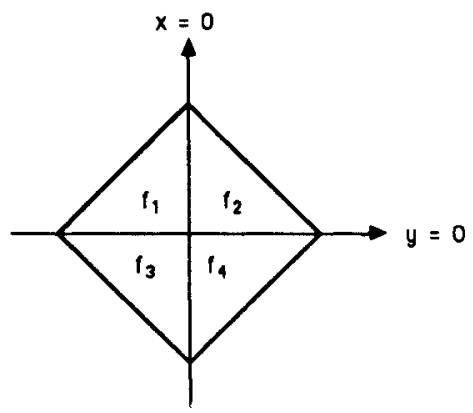

Fig. 2

Proof. Let $t=f_{d}$ and $n=f_{d-1}^{0}$. Then $A(\Delta, r)$ is an $n \times(t+n)$ matrix so $M(\Delta, r) \subset R^{t+n}$. Let $\sigma_{1}, \ldots, \sigma_{t}$ be an ordering of $\Delta_{d}$. Then $F$ in $C^{r}(\Delta)$ can be written $\left(f_{1}, \ldots, f_{t}\right)$ with respect to this ordering. If $\operatorname{dim}\left(\sigma_{i} \cap \sigma_{j}\right)=d-1$, let $l_{i j}$ be the affine form which defines aff $\left(\sigma_{i} \cap \sigma_{j}\right)$. Then

$$
\begin{aligned}
& F \text { is in } C^{r}(\Delta) \Leftrightarrow \quad \text { whenever } \operatorname{dim}\left(\sigma_{i} \cap \sigma_{j}\right)=d-1, f_{i}-f_{j} \in\left(\tilde{l}_{i j}\right) \\
& \Leftrightarrow \quad \text { whenever } \operatorname{dim}\left(\sigma_{i} \cap \sigma_{j}\right)=d-1 \text {, there exists } h_{\tau} \in R \\
& \text { where } \tau=\sigma_{i} \cap \sigma_{j} \text {, such that } f_{i}-f_{j}+h_{t} \tilde{l}_{i j}=0 \\
& \Leftrightarrow \quad\left(f_{1}, \ldots, f_{t}, h_{r_{1}}, \ldots, h_{\tau_{n}}\right) \in M(\Delta, r) \text {, where } \tau_{1}, \ldots, \tau_{n} \text { is an } \\
& \text { ordering of } \Delta_{d-1}^{\circ} \text {. }
\end{aligned}
$$

Example. Let $\Delta$ be the triangulated square shown in Fig. 2. We may write $F \in C^{r}(\Delta)$ as $\left(f_{1}, f_{2}, f_{3}, f_{4}\right)$ as shown. Then $l_{12}=l_{34}=x$ and $l_{23}=l_{14}=y$, so $A(\Delta, r)$ is the following matrix, where $\tilde{x}=x^{r+1}$ and $\tilde{y}=y^{r+1}$;

$$
\left[\begin{array}{rrrr|rrrr}
1 & -1 & 0 & 0 & \tilde{x} & 0 & 0 & 0 \\
0 & 1 & -1 & 0 & 0 & \tilde{y} & 0 & 0 \\
0 & 0 & 1 & -1 & 0 & 0 & \tilde{x} & 0 \\
-1 & 0 & 0 & 1 & 0 & 0 & 0 & \tilde{y}
\end{array}\right]
$$

Let $\hat{\Delta}$ be the homogenization of $\Delta$ as defined in Section 2. Recall that, for $\sigma$ in $\Delta$, $\hat{\sigma}$ denotes the convex hull of $\sigma$ and $v$ denotes the vertex joined to $\Delta$.

Lemma 4.4. If $\Delta \subset \mathbb{R}^{d}$ is hereditary, then $\hat{\Delta} \subset \mathbb{R}^{d+1}$ is also hereditary and $A(\hat{\Delta}, r)={ }^{\mathrm{h}} A(\Delta, r)$, the homogenization of the matrix $A(\Delta, r)$.

Proof. It is immediate that $G(\Delta)=G(\hat{\Delta})$ and so $\partial(\Delta)=\partial(\hat{\Delta})$. Further, since $\mathrm{st}_{\bar{\Delta}}(\hat{\sigma})=\mathrm{st}_{\hat{\Delta}}(\sigma)=\mathrm{st}_{\Delta}(\sigma) \cdot v$, we have that $\hat{\Delta}$ is hereditary.

The columns of $\partial(\hat{\Delta})=\partial(\Delta)$ are homogeneous, so to show that $A(\hat{\Delta}, r)=$ ${ }^{\mathrm{h}} A(\Delta, r)$ we must show that if $l$ is the affine form in $R$ defining $\sigma \in \Delta_{d-1}^{0}$, then ${ }^{\mathrm{b}} l$ is the (linear) form in $R[z]$ defining $\hat{\sigma}$. But this follows from Lemma 2.2 . 
If $\Delta$ is a hereditary $d$-complex, the isomorphism from $M(\hat{\Delta}, r)$ to $C^{r}(\hat{\Delta})$ will be a graded isomorphism since the map is just the restriction of the projection map on $R^{t+k}$ to $M(\hat{\Delta}, r)$. Then $P\left(C^{r}(\hat{\Delta}), \lambda\right)=P(M, \lambda)$ where $M=M(\hat{\Delta}, r)$, and we have the following from Theorem 2.8 and the results of Section 3.

Theorem 4.5. Let $\Delta$ be a hereditary d-complex. Let $r$ be in $\mathbb{N}$. If

$$
\sum \operatorname{dim}_{\mathbb{R}} C_{k}^{r}(\Delta) \lambda^{k}=P(\lambda) /(1-\lambda)^{d+1},
$$

where $P(\lambda)$ is a polynomial in $\lambda$ with integer coefficients, then

(a) $P(1)=f_{d}(\Delta)$,

(b) $P^{\prime}(1)=(r+1) f_{d-1}^{\circ}(\Delta)$, and

(c) $P(\lambda)=t+f_{d-1}^{\circ}(\Delta) \lambda^{r+1}-P(\operatorname{Im} A(\Delta, r), \lambda)$.

Proof. Let $\hat{\Delta}$ denote the homogenization of $\Delta$. Then $P(\lambda)=P\left(C^{r}(\hat{\Delta}), \lambda\right)$, and we must show that $A(\hat{\Delta}, r)$ satisfies conditions (i)-(iii) from Section 3 . Recall that $A(\hat{\Delta}, r)$ is the matrix

$$
\left[\partial \mid \begin{array}{lll}
\tilde{l}_{1} & & \\
& \ddots & \\
& & \tilde{l}_{n}
\end{array}\right] .
$$

It is immediate that $A(\widehat{\Delta}, r)$ satisfies conditions (i) and (ii). To see that it also satisfies (iii), recall that the columns of $\partial(\Delta)$ correspond to $d$-faces of $\Delta$ and the rows correspond to interior $(d-1)$-faces of $\Delta$. Suppose $l_{i}=l_{j}$ and both $\sigma_{i}$ and $\sigma_{j}$ in $\Delta_{d-1}^{\circ}$ lie on some $\sigma$ in $\Delta_{d}$. Since $\sigma$ is a polytope, $Z\left(l_{i}\right)$ and $Z\left(l_{j}\right)$ are supporting hyperplanes for $\sigma_{i}$ and $\sigma_{j}$ in $\sigma$, i.e., $Z\left(l_{i}\right) \cap \sigma=\sigma_{i}$ and $Z\left(l_{j}\right) \cap \sigma=\sigma_{j}$. But $l_{i}=l_{j}$ implies $Z\left(l_{i}\right)=Z\left(l_{j}\right)$ so we must have $\sigma_{i}=\sigma_{j}$. Let $\partial(\Delta)=\left(a_{i k}\right)$. We have shown that if $a_{i k} \neq 0$, and if $l_{i}=l_{j}$, then $a_{j k}=0$ if $j \neq i$. Finally, since $\Delta$ is pure, for every $i$ there is some $k$ such that $a_{i k}$ is nonzero. The $k$ th column will be $B(i)$ as in (iii). The proof now follows from Proposition 3.4, Corollary 3.6, and Theorem 3.9.

Example. For the triangulated square in Fig. 2, we get $P(\lambda)=1+2 \lambda^{r+1}+\lambda^{2 r+2}$. If the edge lying on the positive $y$ axis is perturbed somewhat, then, for $r=1$, $P(\lambda)=1+\lambda^{2}+2 \lambda^{3}$. This shows that $P^{\prime \prime}(1)$ is not given by combinatorial data. See [8] for more examples.

The next application will be to modules arising from arrangements of hyperplanes. Let $K$ be an arbitrary field. A hyperplane arrangement is a set of hyperplanes in $K^{d}$ containing the origin, i.e., linear subspaces of codimension one. Let $X=\left\{H_{1}, \ldots, H_{n}\right\}$ be a hyperplane arrangement and let $l_{1}, \ldots, l_{n}$ denote linear forms defining $H_{1}, \ldots, H_{n}$. We can define

$$
\operatorname{Der}(X) \equiv\left\{F \in R^{d}: l_{i} \mid l_{i} \circ F \text { for all } 1 \leq i \leq n\right\},
$$


where $F$ is thought of as a polynomial map from $K^{d}$ to $K^{d}$ and o denotes the composition of maps. $\operatorname{Der}(X)$ becomes an $R$-module in the obvious way. More generally, a multiarrangement $\tilde{X}$ is a multiset $\left\{H_{1}^{k_{1}}, \ldots, H_{n}^{k_{n}}\right\}$ where $k_{i} \in \mathbb{N}$. Let $\tilde{l}_{i}$ denote $l_{i}^{k_{i}}$. We define

$$
\operatorname{Der}(\tilde{X}) \equiv\left\{F \in R^{d}: \tilde{l}_{i} \mid l_{i} \circ F \text { for all } 1 \leq i \leq n\right\} .
$$

An arrangement $\tilde{X}$ is free if $\operatorname{Der}(\tilde{X})$ is a free $R$-module. The study of free arrangements was initiated in [22] and [31]. For a more algebraic and combinatorial approach see [34] or [36], where multiarrangements were first considered. It turns out that $\operatorname{Der}(X)$ is isomorphic (as a graded $R$-module) to the kernel of the map $A(\tilde{X}): R^{d+n} \rightarrow R^{n}$ given by the matrix

$$
\left[\begin{array}{l|lll} 
& \tilde{l}_{1} & & \\
& \ddots & \\
& & \tilde{l}_{n}
\end{array}\right],
$$

where $B$ is the matrix with entries $b_{i j}$ where $l_{i}=\sum b_{i j} x_{j}$. Since the $l_{i}$ 's are distinct, it is easy to see that $A(\tilde{X})$ satisfies conditions (i), (ii), and (iii). Condition (iii) is trivially satisfied in this case. We can now apply Proposition 3.4 , Corollary 3.6 , and Theorem 3.9 to get the following result.

Theorem 4.6. Let $\tilde{X}=\left\{H_{1}^{k_{1}}, \ldots, H_{n}^{k_{n}}\right\}$ be a multiarrangement of hyperplanes. Then $\operatorname{Der}(\tilde{X})$ has rank $d$ and if $P(\lambda)=P(\operatorname{Der}(\tilde{X}), \lambda)$, we have

(a) $P(1)=d$,

(b) $P^{\prime}(1)=\sum_{i=1}^{n} k_{i}$, and

(c) $P(\lambda)=t+\sum_{j=1}^{n} \lambda^{d_{j}}-P(\operatorname{Im} A(\tilde{X}), \lambda)$.

Again $P^{\prime \prime}(1)$ is not given by combinatorial data [34, Example 4.2.7]. However, for simple arrangements (each $\left.k_{i}=1\right) P^{\prime \prime}(1)$ is a combinatorial invariant [21]. In the case where $\operatorname{Der}(\tilde{X})$ is a free $R$-module, the proof of $(b)$ is simpler. (See [22] and [36].)

\section{Computational Techniques}

In this section we give a summary of the concept of Grobner bases for ideals and modules over polynomial rings. These "bases" are generating sets which have properties useful for computation. The algorithm for constructing Gröbner bases, due to Buchberger (see, for example, [9]), is a generalization of the division algorithm for polynomials of one variable. In particular, using Gröbner bases we can solve systems of polynomial equations, i.e., we can do linear algebra over polynomial rings. For example, let $R=K\left[x_{1}, \ldots, x_{d}\right]$ where $K$ is a field and let $A$ be an $m \times t$ matrix with entries in $R$. The technique of Gröbner bases can be used to find all $t$-tuples $f=\left(f_{1}, \ldots, f_{t}\right)$ in $R^{t}$ so that $A f=0$. This means we can compute 
a generating set for the kernel of $A$, viewed as a map from $R^{1}$ to $R^{m}$. But this is precisely what we want in the case of splines. Recall from Section 3 that $C^{r}(\Delta)$ is isomorphic to $M=M(\Delta, r)$ where $M$ was the kernel of the map $A=A(\Delta, r)$.

We now describe the theory of Gröbner bases and show how it can be used to compute dimensions of the $\mathbb{R}$-spaces $C_{m}^{r}(\Delta)$ as well as explicit bases for these vector spaces over $\mathbb{R}$.

In order to compute effectively in polynomial rings we need a linear ordering of the monomials which refiness the usual partial order (ordering by divisibility). Let $R=K\left[x_{1}, \ldots, x_{n}\right]$ be the polynomial ring in $n$ variables over $K$, where $K$ is a field. Let $T$ be the set of all monomials in $R$.

Definition. A multiplicative order $>$ on $R$ is a total order on $T$ such that:

(i) $m>1$ for all monomials $m$ in $T$.

(ii) If $m, n \in T$ and $m>n$, then, for all $\alpha \in T, \alpha m>\alpha n$.

The graded lexicographic order is a multiplicative order which we will find most useful. It is defined by $x_{1}^{a_{1}} \cdots x_{n}^{a_{n}}>x_{1}^{b_{1}} \cdots x_{n}^{b_{n}}$ if and only if $a_{1}+\cdots+a_{n}>b_{1}+$ $\cdots+b_{n}$ or $a_{1}+\cdots+a_{n}=b_{1}+\cdots b_{n}$ and $a_{i}>b_{i}$ for the smallest $i$ such that $a_{i} \neq b_{i}$. For more about multiplicative orderings (also called term orderings), see [19].

Given an ordering $>$ and an element $f$ in $R$, we define the initial term of $f$, In $(f)$, to be the largest monomial under $>$ which appears in $f$. Let $S \subset R$ be any set. We define the initial ideal of $S, \operatorname{In}(S)$, to be the ideal generated by the initial terms of elements of $S$.

We can extend this notion to free modules over $R$ of finite rank $t$, viewed as $t$ tuples of elements in $R$. A monomial in $R^{t}$ has the form $m e_{i}$ where $m$ is a monomial in $R$ and $e_{i}$ is the ith standard basis vector of $R^{t}$. We can also write $(0, \ldots, 0, m, 0, \ldots, 0)$ where the $m$ is in the $i$ th place. The ordering here will be defined by $m e_{i}>n e_{j}$ if and only if $i>j$ or $i=j$ and $m>n$ under the given multiplicative ordering on $R$. If $S \subset R^{t}$, then $\operatorname{In}(S)$ will be the submodule of $R^{t}$ generated by the initial terms of elements of $S$.

Proposition 5.1. Let $M$ be a submodule of $R^{t}$. (If $t=1$, this is just an ideal.) $A$ set $G=\left\{G_{1}, \ldots, G_{k}\right\}$ in $M$ is called a Gröbner basis for $M$ if any of the following equivalent conditions hold:

(1) $\operatorname{In}(G)=\operatorname{In}(M)$.

(2) If $F$ is in $M$, then there exist $r_{1}, \ldots, r_{k}$ in $R$ so that $F=\sum_{i=1}^{k} r_{i} G_{i}$, and for all $i$, $\operatorname{In}\left(r_{i} G_{i}\right) \leq \operatorname{In}(F)$.

(3) The set $\Gamma=\left\{m G_{i}: m\right.$ is a monomial in $x_{1}, \ldots, x_{d}$, and $\operatorname{In}\left(G_{j}\right)$ does not divide $\operatorname{In}\left(m G_{i}\right)$ for all $\left.j<i\right\}$ is a vector space basis for $M$ over $K$.

The equivalence of these conditions can be found in [30], who credit Bayer [5] and Lazard [15]. It follows from (2) that $G$ generates $M$. We will see that when $M$ is graded, the first characterization is useful for computing Hilbert series and the third for finding vector space bases for the $i$ th graded piece, $M_{i}$, over $K$. 
We now describe how the theory of Gröbner bases relates to the spline modules $C^{r}(\Delta)$. To do this we restrict ourselves to hereditary complexes in order to use the characterization of $C^{r}(\Delta)$ from Section 4. If we have a Gröbner basis for $C^{r}(\Delta)$, and $\Gamma$ is as in (3) in the above proposition, then the set $\Gamma_{k}=\{\gamma \in \Gamma:$ degree $\gamma \leq k\}$ will be an $\mathbb{R}$-basis for $C_{k}^{r}(\Delta)$. This will be true because of the graded nature of the ordering we have chosen.

Let $\Delta$ be a hereditary complex. Recall the setup from Section 3 . We have the exact sequence

$$
0 \longrightarrow M \longrightarrow R^{t+n} \stackrel{A}{\longrightarrow} R^{n} \longrightarrow N \longrightarrow 0
$$

where $N$ is the cokernel of $A$. Then $M$ consists of all syzygies of the columns of $A$. This means that if $F_{1}, \ldots, F_{t+n}$ are the columns of $A$, then $\left(g_{1}, \ldots, g_{t+n}\right) \in M$ (we may think of $M$ as sitting inside of $R^{t+n}$ ) if and only if $F_{1} g_{1}+F_{2} g_{2}+\cdots+$ $F_{t+n} g_{t+n}=0$ in $R^{n}$. Using algorithms due to Spear [25], Schreyer [23], and Buchberger [9], we can construct a Gröbner basis for $M$. (See also Proposition 2.11 of [5].) For further discussion of these algorithms and specific examples, see [8].

Now we discuss a method for computing Hilbert series without computing any syzygy modules. In Section 1 we saw that if we can compute the Hilbert series of $C^{r}(\hat{\Delta})$, this will give us the dimensions of the $C_{k}^{r}(\Delta)$ 's as $\mathbb{R}$-vector spaces for all $k$. It turns out that we can compute the Hilbert series of $C^{r}(\hat{\Delta})$ (which is exactly the dimension series for $C_{k}^{r}(\Delta)$ ), without actually finding a Gröbner basis for $C^{r}(\hat{\Delta})$. From Corollary 3.6 we have the formula

$$
P(\lambda)=f_{d}(\Delta)+f_{d-1}^{\circ}(\Delta) \lambda^{r+1}-P(\operatorname{Im} A(\Delta, r), \lambda)
$$

where the Hilbert series of $C^{r}(\hat{\Delta})$ (and thus the dimension series of $C_{k}^{r}(\Delta)$ ) has the form $P(\lambda) /(1-\lambda)^{d+1}$. We have now reduced the original problem to finding the Hilbert series of the image of $A(\Delta, r)$. But we know a generating set for this module, namely the columns of $A(\Delta, r)$, so we can compute a Gröbner basis for it. The following proposition, originally proved for ideals in [16], allows us to reduce the problem to an even simpler situation.

Proposition 5.2. If $G$ is a Gröbner basis for a module $N$ and $H$ denotes the Hilbert function of $N$, then $H(N)=H(\operatorname{In}(N))$.

By (1) of Proposition 5.1, this gives $H(N)=H(\operatorname{In} G)$. We have thus reduced the problem to that of computing the Hilbert series of a monomial module, $\operatorname{In}(G)$. This is basically an inclusion-exclusion calculation.

For more details about the nature of these computations, see [8].

\section{Acknowledgments}

The authors are grateful to Michael Stillman for pointing out an error in a preliminary version of this work, and to Bernd Sturmfels and Günter Ziegler for many constructive suggestions. 


\section{References}

1. P. Alfeld, On the dimension of multivariate piecewise polynomial functions, in Proceedings of the Biennial Dundee Conference on Numerical Analysis, Pitman, London, 1985.

2. P. Alfeld, B. Piper, and L. L. Schumaker, An explicit basis for $C^{1}$ quartic bivariate splines, SIAM J. Numer. Anal. 24 (1987), 891-911.

3. P. Alfeld and L. L. Schumaker, The dimension of bivariate spline spaces of smoothness $r$ for degree $d \geq 4 r+1$, Constr. Approx. 3 (1987), 189-197.

4. M. F. Atiyah and I. G. Macdonald, Introduction to Commutative Algebra, Addison-Wesley, Reading, MA, 1969.

5. D. A. Bayer, The Division Algorithm and the Hilbert Scheme, Ph.D. Dissertation, Harvard University, 1982.

6. L. J. Billera, The algebra of continuous piecewise polynomials, Adv. in Math. 76 (1989), 170-183.

7. L. J. Billera, Homology of smooth splines: generic triangulations and a conjecture of Strang, Trans. Amer. Math. Soc. 310 (1988) 325-340.

8. L. J. Billera and L. L. Rose, Gröbner basis methods for multivariate splines, in Mathematical Methods in Computer Aided Geometric Design, T. Lyche and L. L. Schumaker, eds., Academic Press, New York, 1989, pp. 93-104.

9. B. Buchberger, Gröbner bases: An algorithmic method in polynomial ideal theory, in Multidimensional Systems Theory, N. K. Bose, ed., Reidel, Dordrecht, 1985, pp. 184-232.

10. C. K. Chui and R. H. Wang, On smooth multivariate spline functions, Math. Comp. 41 (1983), 131-142.

11. H. Crapo and J. Ryan, Spatial realizations of linear scenes, Structural Topology 13 (1986), 33-68.

12. B. Grünbaum, Convex Polytopes, Wiley-Interscience, London, 1967.

13. R. Hartshorne, Algebraic Geometry, Spring-Verlag, New York, 1977.

14. I. Kaplansky, Commutative Rings, University of Chicago Press, Chicago, IL, 1974.

15. D. Lazard, Gröbner bases, Gaussian elimination, and resolution of systems of algebraic equations, in Computer Algebra, Proceedings EU ROCAL'83, J. A. van Hulzen, ed., Lecture Notes in Computer Science, Vol. 162, Springer-Verlag, New York, 1983, pp. 146-156.

16. F. S. Macaulay, Some properties of enumeration in the theory of modular systems, Proc. London Math. Soc. 26 (1927), 531-555.

17. H. Matsumura, Commutative Algebra, Second Edition, Benjamin, London, 1980.

18. P. Orlik, Introduction to Arrangements, CBMS Regional Research Conference Series, No. 72 , American Mathematical Society, Providence, RI, 1989.

19. L. Robbiano, Term orderings on the polynomial ring, Proceedings of EUROCAL 85, Lecture Notes in Computer Science, Vol. 204, Spring-Verlag, New York, 1985, pp. 513-517.

20. L. L. Rose, The Structure of Modules of Splines over Polynomial Rings, Ph.D. Thesis, Cornell University, Ithaca, NY, January, 1988.

21. L. L. Rose and H. Terao, Hilbert polynomials and geometric lattices, $A d v$. in Math. (to appear).

22. K. Saito, Theory of logarithmic differential forms and logarithmic vector fields, J. Fac. Sci. Univ. Tokyo Sect. IA 27 (1980), 265-291.

23. F.O. Schreyer, Die Berechnung von Syzygien mit dem verallgemeinerten Weierstraßschen Divisionsatz und eine Anwendung auf analytische Cohen-Macaulay, Stellenalgebren minimaler Multiplizität, Diplomarbeit am Fachbereich Mathematik der Universitat Hamburg, 1980.

24. L. Solomon and $\mathrm{H}$. Terao, A formula for the characteristic polynomial of an arrangement, $A d v$. in Math. 64 (1987), 305-325.

25. D. A. Spear, A constructive approach to commutative ring theory, Proceedings of the 1977 MACSYMA Users' Conference, NASA CP-2012, National Technical Information Service, Springfield, VA, 1977, pp. 369-376.

26. R. P. Stanley, Combinatorics and Commutative Algebra, Birkhauser, Boston, 1983.

27. R. P. Stanley, Enumerative Combinatorics, Vol. I, Wadsworth \& Brooks/Cole, Monterey, CA, 1986.

28. P. F. Stiller, Certain reflexive sheaves on $P_{C}^{n}$ and a problem in approximation theory, Trans. Amer. Math. Soc. 279 (1983), 125-142.

29. P. F. Stiller, Vector bundles on complex projective spaces and systems of partial differential equations, I, Trans. Amer. Math. Soc. 298 (1986), 537-548. 
30. B. Sturmfels and N. White, Gröbner bases and invariant theory, Adv. in Math. 76 (1989), 245-259.

31. H. Terao, Arrangements of hyperplanes and their freeness, I, J. Fac. Sci. Univ. Tokyo Sect. IA 27 (1980), 293-312.

32. W. Whiteley, Realizability of polyhedra, Structural Topology 1 (1979), 46-58.

33. O. Zariski and P. Samuel, Commutative Algebra, Vol. II, Van Nostrand, Princeton, NJ, 1960; Springer-Verlag, New York, 1975.

34. G. M. Ziegler, Algebraic Combinatorics of Hyperplane Arrangements, Ph.D. Thesis, Massachusetts Institute of Technology, Cambridge, MA, May, 1987.

35. G. M. Ziegler, Combinatorial construction of logarithmic differential forms, Adv. in Math. 76 (1989), 116-154.

36. G. M. Ziegler, Multiarrangements of hyperplanes and their freeness, in Proceedings, International Conference on Singularities, Iowa City, Iowa, 1986, R. Randell, ed., Contemporary Mathematics, Vol. 90, American Mathematical Society, Providence, RI, 1989.

Received August 16, 1988, and in revised form May 24, 1989. 